\title{
Determination of physical properties of the asteroid (41) Daphne from interferometric observations in the thermal infrared ts
}

\author{
Alexis Matter ${ }^{\mathrm{a}, 1, *}$, Marco Delbo $^{\mathrm{b}}$, Sebastiano Ligori ${ }^{\mathrm{c}}$, Nicolas Crouzet $^{\mathrm{b}, 2}$, \\ Paolo Tanga ${ }^{\mathrm{b}}$ \\ ${ }^{a}$ UNS-CNRS-Observatoire de la Côte d'Azur, Laboratoire Fizeau, BP 422906304 Nice \\ cedex 04 , France. \\ ${ }^{b}$ UNS-CNRS-Observatoire de la Côte d'Azur, Laboratoire Cassiopée, BP 422906304 Nice \\ cedex 04 , France. \\ ${ }^{c}$ INAF-Osservatorio Astronomico di Torino, Strada Osservatorio 20, 10025 Pino Torinese, \\ Torino, Italy.
}

Number of pages: 44

Number of tables: 3

Number of figures: 8

\footnotetext{
${ }^{2}$ Based on observations collected at the European Southern Observatory (ESO), Chile: ESO Program ID 080.C-0195, VISA-Italy.

${ }^{*}$ Corresponding author.

Email address: amatter@mpifr-bonn.mpg.de (Alexis Matter)

${ }^{1}$ Present address: Max-Planck Institut für Radioastronomie, Auf dem Hügel 69, 53121 Bonn, Germany.

${ }^{2}$ Present address: Space Telescope Science Institute, 3700 San Martin Drive, Baltimore, MD 21218, USA.
} 
Proposed running head: Asteroid interferometry in the thermal IR.

Editorial correspondence and proofs should be directed to:
Alexis Matter
Max-Planck Institut für Radioastronomie,
Auf dem Hügel 69, 53121 Bonn, Germany.
email: amatter@mpifr-bonn.mpg.de;
Tel: +49228525186
Fax: +49228 525229 


\begin{abstract}
We describe interferometric observations of the asteroid (41) Daphne in the thermal infrared obtained with the Mid-Infrared Interferometric Instrument 3 (MIDI) and the Auxiliary Telescopes (ATs) of the European Southern Obser4 vatory (ESO) Very Large Telescope Interferometer (VLTI). We derived the size and the surface thermal properties of (41) Daphne by means of a thermophysical model (TPM), which is used for the interpretation of interferometric data for the first time. From our TPM analysis, we derived a volume equivalent diameter for (41) Daphne of $189 \mathrm{~km}$, using a non-convex 3-D shape model derived from optical lightcurves and adaptive optics images (B. Carry, private communication). ${ }_{10}$ On the other hand, when using the convex shape of Kaasalainen et al. (2002.

11 Icarus 159, 369-395) in our TPM analysis, the resulting volume equivalent diameter of (41) Daphne is between 194 and $209 \mathrm{~km}$, depending on the surface roughness. The shape of the asteroid is used as an a priori information in our 14 TPM analysis. No attempt is made to adjust the shape to the data. Only the 15 size of the asteroid and its thermal parameters such as, albedo, thermal inertia and roughness are adjusted to the data. We estimated our model systematic uncertainty to be of $4 \%$ and of $7 \%$ on the determination of the asteroid volume equivalent diameter depending on whether the non-convex or the convex shape 9 is used, respectively. In terms of thermal properties, we derived a value of the surface thermal inertia smaller than $50 \mathrm{~J} \mathrm{~m}^{-2} \mathrm{~s}^{-0.5} \mathrm{~K}^{-1}$ and preferably in the range between 0 and $\sim 30 \mathrm{~J} \mathrm{~m}^{-2} \mathrm{~s}^{-0.5} \mathrm{~K}^{-1}$. Our TPM analysis also shows
\end{abstract}




\section{Keywords}

${ }_{24}$ Asteroids; Asteroids surfaces; Infrared observations; Data reduction techniques. 


\section{Introduction}

Information about sizes and shapes of asteroids provides essential constraints to the history and formation processes of these bodies (Bottke et al., 2005; Tanga et al., 2009). The size distribution of the different subpopulations of asteroids and of the asteroid dynamical families constrain the collisional evolution processes that these bodies have experienced during their history (Bottke et al., 2005). Moreover, accurate determination of sizes and shapes is crucial to estimate volumes of asteroids, which allow one to calculate the bulk densities of these bodies when their masses are determined by some means. The density and internal structure are among the most important characteristics of asteroids, yet they are also some of the least constrained. When compared with the densities of meteorites - a partial sample of the building blocks of asteroids that survive the passage through the Earth's atmosphere - one can deduce the nature of asteroid interiors. These physical properties of asteroids reflect the accretional and collisional environment of the early solar system.

The determination of the volumes of asteroids will be particularly important in the next future when more asteroid masses are expected to be accurately derived. For instance, it has been estimated that the masses of slightly more than 100 asteroids will be determined to better than $30 \%$ (relative accuracy) from the gravitational perturbations that these bodies exert on the orbits of smaller asteroids thanks to the high accuracy astrometric measurements of the ESA space mission Gaia (launch in 2013; Mouret et al., 2007). Yet, the volume of these bodies are not known with accuracies small enough to allow one to calculate meaningful densities. The volumes of asteroids are affected by large errors because their true 3-dimensional shapes are generally unknown and thus approximated by means of spheres. It can be noted, for instance that by using a shape and a spin solution derived from lightcurves and mutual occultation events for the asteroid (22) Kalliope, Descamps et al. (2008) significantly revised its volume and thus its density compared to previous estimates based on a sphere. The error in the estimation of the volume can also be significant when 
large scale topographic concavities, known to be present on asteroids (see e.g., Thomas et al., 1999, 2002), are approximated with flat surfaces.

From the size $(D)$ and the absolute magnitude of an asteroid in the visible light $(H)$, one can derive the geometric visible albedo $\left(p_{V}\right)$ using the formula:

$$
D(k m)=1329 p_{V}^{-1 / 2} 10^{-H / 5}
$$

The value of the albedo is important to constrain the nature of asteroids: it is known, for instance, that asteroids with spectra similar to carbonaceous chondrite meteorites (types CI and CM), the so-called C-type asteroids, have values of $p_{V}$ between 0.03 and 0.10 (Stuart and Binzel, 2004); stony (S-type) asteroids, rich in silicates such as olivine and pyroxene have moderate values of $p_{V}$ (e.g., between $\sim 0.15$ and $\sim 0.3$ ) (Stuart and Binzel, 2004), whereas asteroids whose reflectance spectrum is analog to that of enstatite meteorites are known to have in general high $(>0.4)$ albedo values (see e.g., Tedesco et al., 1989, and references therein). In general sizes and albedos of asteroids are obtained form photometric observations of these bodies in the thermal infrared (see Harris and Lagerros, 2002, for a review on the topic). Models of the surface temperature distribution and the corresponding infrared emission are used for the analysis of observational data (Delbo and Harris, 2002; Harris and Lagerros, 2002). In particular, thermophysical models (TPM) take explicitly into account the effects of thermal inertia, spin state, asteroid shape and surface roughness on the calculation of asteroids infrared emission.

One of these parameters, the thermal inertia, a measure of the resistance of a material to temperature change, is particularly important. It is defined by $\Gamma=\sqrt{\rho \kappa c}$, where $\kappa$ is the thermal conductivity, $\rho$ the density and $c$ the specific heat. The value of the thermal inertia depends on the material properties (see Mueller, 2007, and references therein for a table of thermal inertia values of some typical materials) and inform us about the nature of the surface regolith: a soil with a very low value of $\Gamma$, for instance in the range between 20 and 50 $J m^{-2} s^{-0.5} K^{-1}$, is covered with fine dust; an intermediate value (150-700 
$\left.J \mathrm{~m}^{-2} \mathrm{~s}^{-0.5} \mathrm{~K}^{-1}\right)$ indicates a coarser, mm- to $\mathrm{cm}$-sized, regolith as observed on (433) Eros (Veverka et al., 2001a,b) and (25143) Itokawa (Yano et al., 2006), respectively; solid rock with very little porosity is known to have thermal inertia values of more than $2500 \mathrm{~J} \mathrm{~m}^{-2} \mathrm{~s}^{-0.5} \mathrm{~K}^{-1}$ (Jakoskv, 1986). The correlation between the value of $\Gamma$ and the nature of the soil has been also demonstrated from study of the martian surface (see e.g., Christensen et al., 2003). Moreover, because thermal inertia controls the surface temperature distribution of an asteroid, it affects the strength of the Yarkovsky effect. This is the gradual drifting of the semi major axis of the orbits of km-sized asteroids caused by the asymmetric (with respect to the direction asteroid-sun) emission of the thermal infrared radiation that carry momentum (see Bottke et al., 2006, and references therein). This effect plays a role in the delivery of near-Earth asteroids from the main belt (Morbidelli and Vokrouhlický, 2003), in the dispersion of asteroid families (Nesvorný and Bottke, 2004), and it is a major source of uncertainty in the impact prediction estimations for potentially hazardous asteroids (Giorgini et al., 2002; Milani et al., 2009). Finally, accurate determination of thermal inertia is important in the estimation of systematic errors on sizes and albedos of asteroids, when these parameters are determined by means of simple thermal models (see e.g., Spencer et al., 1989).

As shown by Delbo et al. (2009), the Very Large Telescope Interferometer (VLTI) of the European Southern Observatory (ESO) can be used to obtain measurements of asteroid sizes and shapes. Generally speaking, the VLTI has the ability of measuring directly sizes and deriving rough information about the shape of asteroids from measurements of the visibility (contrast) of interferometric fringes. The visibility is a function of the apparent angular extension of the body along the projected interferometer baseline. Shape features such as large concavities, bilobed shapes and/or presence of satellites, also produce a clear signature in the visibility. A sensitive instrument to measure asteroid visibilities at the VLTI in the mid-infrared N-band $(8-13 \mu \mathrm{m})$ is the Mid-Infrared Interferometric Instrument (MIDI; Leinert et al., 2003). The angular resolv- 
ing power of the interferometer depends on the length of the baseline. VLTI baselines vary between 16 and $130 \mathrm{~m}$, with theoretical corresponding angular resolutions between 130 and 16 mas (milliarcseconds) at $10 \mu \mathrm{m}$.

Interferometric observations of asteroids with other facilities, such as the Fine Guidance Sensors (FGS) of the Hubble Space Telescope (HST, Tanga et al., 2001; Hestroffer et al., 2002; Tanga et al., 2003), demonstrated the capability of the method of obtaining sizes of asteroids and reconstructing the ellipsoids that best fit their shape. Given the limiting magnitude of the FGS, only bright $(\mathrm{V} \leq 12)$ and large $(\sim 100 \mathrm{~km}$-sized) asteroids were observed by this program. However, only the large VLTI baselines can overcome these sensitivity and size limitations by extending the use of the interferometric technique to a large number of fainter and smaller targets. One particularly interesting feature of the MIDI instrument is that it also measures the total (non coherent) spectral energy distribution, $I(\lambda)$, of the source in the 8-13 $\mu \mathrm{m}$ spectral interval. This thermal infrared data can then be used as a further constraint to derive asteroid sizes, through the application of asteroid thermal models. In their work, Delbo et al. (2009) show the first successful interferometric observations of two asteroids with MIDI, (234) Barbara and (951) Gaspra.

In this work, we report on the continuation of the program devoted to measurement of the physical properties of asteroids from interferometric observations in the thermal infrared. In particular, we obtained the first successful interferometric observations of asteroids using the Auxiliary Telescopes (ATs) of the VLTI. From fluxes and interferometric visibilities measured in the thermal infrared, we derived the size of the asteroid (41) Daphne, and we studied its thermal properties by means of a TPM.

This work is structured as follows: in section 2 we detail the thermophysical model used for the interpretation of MIDI data in terms of asteroid physical properties; in section 3 we report the observations and the data reduction process that we adopted; in section 4, we detail the shape models that we used; in section 5, we give our results, followed by a discussion in section 6 . 


\section{Modeling and analysis of MIDI observations}

MIDI is used to coherently combine the infrared light collected by two of the four 8-m Unit Telescopes (UT) or by two of the four 1.8-m Auxiliary Telescopes (AT) of the ESO VLTI. The two observables measured by MIDI are the photometric flux $I(\lambda)$ and the visibility $V(u, v)$ of the source; where $u=B_{x} / \lambda$ and $v=B_{y} / \lambda$ are the spatial frequencies in $\operatorname{rad}^{-1}$ along the $\mathrm{x}$ - and $\mathrm{y}$-axis, with $B_{x}$ and $B_{y}$ the components, along the two axis, of the interferometer's baseline, projected on the plane of the sky. The $\mathrm{x}$ and $\mathrm{y}$-axis define the coordinates on this plane. We recall that $V(u, v)$ is the Fourier transform of the brightness distribution of the source divided by $I(\lambda)$. The visibility can be also indicated with $V(\mathbf{B} / \lambda)$, where $\mathbf{B}$ is a vector of components $\left(B_{x}, B_{y}\right)$.

Delbo et al. (2009) interpreted $I(\lambda)$ and $V(\mathbf{B} / \lambda)$ using simple thermal models and simple geometric models (disk of uniform brightness and system of two disks) in order to derive the size of (951) Gaspra and (234) Barbara. For the latter asteroid, a disk of uniform brightness poorly reproduced the observations, whereas a binary model provided a good fit to the data. For this reason Delbo et al. (2009) speculated that Barbara has a bilobed shape or a satellite. When we have a priori information about the shape and the spin state of an asteroid, TPMs can be used to derive the size of the body and to constrain surface properties, such as albedo and thermal inertia and macroscopic roughness. These parameters are explicitly taken into account in the TPM to calculate the asteroid's thermal emission, and are adjusted until model fluxes best fit simultaneously observations obtained at different epochs and wavelengths in the thermal infrared. In general, these observations are measurements of the object's disk integrated thermal infrared flux $I(\lambda)$ (see e.g., Müller and Barnes, 2007; Delbo and Tanga, 2009).

Here, we used a TPM to calculate interferometric visibilities of asteroids in the thermal infrared for the first time. Our procedure consists in generating images of the thermal infrared emission of the asteroid at different wavelengths as viewed by the observer and then obtaining the model visibility and flux for 
each image: the model flux is calculated by taking the integral of all pixels at each wavelength, while the model visibility is calculated as the modulus of the Fourier transform of the image, along the projected baseline direction, divided by the flux.

The free parameters of the TPM are adjusted in order to minimize the distance between the disk integrated flux $I^{\prime}(\lambda)$ and visibility $V^{\prime}(\mathbf{B} / \lambda)$ of the model, and the corresponding observed quantities $I(\lambda)$ and $V(\mathbf{B} / \lambda)$. As goodness of the fit indicator, we use the reduced $\chi^{2}$, namely:

$$
\bar{\chi}^{2}=\frac{1}{N}\left[\sum_{i=1}^{N_{e}} \sum_{j=1}^{F_{i}}\left(\frac{I_{i}\left(\lambda_{j}\right)-I_{i}^{\prime}\left(\lambda_{j}\right)}{\sigma_{I_{i, j}}}\right)^{2}+\sum_{i=1}^{N_{E}} \sum_{j=1}^{W_{i}}\left(\frac{V_{i}\left(\mathbf{B} / \lambda_{j}\right)-V_{i}^{\prime}\left(\mathbf{B} / \lambda_{j}\right)}{\sigma_{V_{i, j}}}\right)^{2}\right]
$$

where the indexes $i$ and $j$ run over the observation epochs and the discrete samples in wavelength at which the visibility and the flux were measured, $N_{e}$ and $N_{E}$ are the number of epochs at which flux and visibility are respectively measured, $F_{i}$ and $W_{i}$ are the number of flux and visibility samples at the $\mathrm{i}^{\text {th }}$ epoch, $\quad N=\sum_{i=1}^{N_{e}} N_{\lambda, I}^{i}+\sum_{i=1}^{N_{E}} N_{\lambda, V}^{i}$ is the total number of measurements, and $\sigma_{V_{i, j}}$ and $\sigma_{I_{i, j}}$ are the uncertainties on the measured visibilities and fluxes, respectively.

The physical parameters of our TPM, are:

- An a priori information about the shape of the body, described by a mesh of planar triangular facets and the spin vector of the asteroid. The shape and the spin vector are in general determined by lightcurve inversion (see Kaasalainen et al., 2002, for a review), disk-resolved imaging (from e.g., in-situ, the Hubble Space Telescope, and/or ground-based adaptive optics observations; see e.g., Carry et al., 2010), or radar (see e.g., Ostro et al., 2000). Our implementation of the TPM allows non-convex shapes to be used. Shadowing of facets to the observer due to the body's topography is fully taken into account. Mutual heating and light reflection between facets within topographic concavities are not modeled in this version of the TPM. However, their effect is of second order on the surface temperature 
determination. The spin vector solution is given by the rotational phase $\phi_{0}$ at a reference epoch $t_{0}$, the ecliptic longitude $\lambda_{0}$ and latitude $\beta_{0}$ of the spin axis direction, and the rotation period $P$. Note that $t_{0}$ can be quite far in the past. As a consequence, the absolute rotational phase, $\phi(t)$, of the asteroid, at a more recent epoch (e.g., the time of VLTI observations), can be affected by a significant uncertainty, $\Delta_{\phi}$. The latter depends on the error $\sigma_{P}$ on $P$, the value of $P$, and by how far $t$ is from $t_{0}$. Since $\phi=\phi_{0}+2 \pi\left(t-t_{0}\right) P^{-1}$, we can write that

$$
\Delta_{\phi}=2 \pi\left(t-t_{0}\right) P^{-2} \sigma_{P}
$$

neglecting the error on $\phi_{0}$ (which is safe - in general - to assume small). When $\Delta_{\phi} \gtrsim 10^{\circ}$, then $\Delta_{\phi}$ should be treated as a free parameter of the TPM.

- The size of the body. This is described by a factor $a$ that linearly scales all vertexes of the mesh. We give the size of the body in terms of the diameter of the sphere of equivalent volume $D_{\vee}=2\left(\frac{3 \vee}{4 \pi}\right)^{\frac{1}{3}}$, where $\vee$ is the volume of the mesh.

- The bolometric Bond's albedo $A$. This is related to $p_{V}$ via the relation: $A=p_{V}(0.29+0.684 G)$, where $G$ is the slope parameter of the $H, G$ system of Bowell et al. (1989). Although the value reported in the MPC for (41) Daphne is 0.10, the use of the typical default value, namely $G=0.15$ (Bowell et al., 1989), does not significantly affect our results and we decided to keep it.

- The macroscopic surface roughness. This is modeled by adding hemispherical craters of opening angle, $\gamma_{c}$, and surface density, $\rho_{c}$. Following Delbo and Tanga (2009), we used here four preset combinations of $\gamma_{c}$ and $\rho_{c}$ spanning the range of possible values of surface roughness. These values of macroscopic roughness are given in Table1, including the corresponding value of the mean surface slope, $\bar{\theta}$, as defined by Hapke (1984). 
- The value of thermal inertia, which affects the temperature of each tile of the mesh and the temperature distribution inside craters.

Albedo, thermal inertia, and roughness are assumed constant over the surface of the body.

\section{[Table 1 here]}

In order to compute images of the model thermal emission, the first step is to use the TPM to calculate the body's surface temperature distribution, namely the temperature of each tile of the mesh and inside craters at any given epoch $t$. This is performed by solving the one-dimensional heat diffusion equation for each tile of the mesh (see Mueller, 2007, for more details). The boundary conditions of the heat diffusion equation are given, at the surface, by the input radiative heating from the Sun and irradiation of the heat into space, and, at depth, by imposing a zero net heat flow towards the interior of the body. The position of the Sun (the heat source) with respect to each facet is calculated by applying the inverse coordinates transformations of Durech et al. (2010) in order to transform the heliocentric position vector of the asteroid into the body's mesh reference frame: the transformation is a function of the time $t$. The method we adopt to calculate the temperature distribution inside craters is given by Emery et al. (1998). We do not explicitly model thermal conduction inside craters, and here we use the approximation of Lagerros (1998). This approximation cannot be used on the night side. However, this is not a limitation for the present study because our observations took place at a moderate solar phase angle (see Table 2). Consequently, the fraction of the night side seen by the observer was negligible. Because of the finite thermal inertia value, the heat diffusion is not instantaneous and the body temperature distribution depends on his past illumination history. For this reason, the calculation of the heat diffusion is started $\sim 100$ rotations ( $\sim$ a month) before the observation epochs. We carefully checked that the body temperature distribution stabilizes and is inde- 
pendent of the initial conditions. From the knowledge of the body temperature distribution, model fluxes are calculated assuming a wavelength independent emissivity of 0.9 , for each tile of the mesh, including craters, in the direction towards the observer. The emissivity of 0.9 assumed here is typical for the vast majority of silicate powders. The emissivity of these materials is within $10 \%$ of 0.9 in the wavelength range between 7 and $14 \mu m$ (see e.g., Mueller, 2007, and references therein). Then the three-dimensional mesh is projected on the plane of the sky to create a two dimensional image of the asteroid. In order to have a reasonable image size, while remaining close to the maximum resolution of the MIDI instrument, we sampled the images with a resolution of 4 mas/pixel (5 mas/pixels for the convex shape of 41 Daphne; see section 5). Our results are robust with respect to changes of the pixel scale. The value of each pixel is calculated from the flux of the facet of the mesh on which the pixel is projected to: namely it is the facet flux multiplied by the area of the pixel and divided by the projected area of the facet. Fig. 1 shows two images of (41) Daphne obtained along the lines described above, from the two shape models described later. These images are created for each wavelength.

[Fig. 1 here]

Then the integrated flux and visibility of the model are given by:

$$
\begin{gathered}
I^{\prime}\left(\lambda_{j}\right)=a^{2} \sum_{x, y} O^{\prime}\left(x, y, \lambda_{j}\right) \\
V^{\prime}\left(\frac{B}{\lambda_{j}}\right)=\frac{F T\left(O^{\prime}\left(\frac{x^{\prime}}{a}, \frac{y^{\prime}}{a}, \lambda_{j}\right)\right)}{I^{\prime}\left(\lambda_{j}\right)}=\frac{a \hat{O}^{\prime}\left(\frac{a B}{\lambda_{j}}\right)}{I^{\prime}\left(\lambda_{j}\right)} \\
=\frac{a}{I^{\prime}\left(\lambda_{j}\right)} \sum_{y^{\prime}}\left(\sum_{x^{\prime}} O^{\prime}\left(x^{\prime}, y^{\prime}, \lambda_{j}\right)\right) e^{\frac{-i 2 \pi a B}{N_{y^{\prime} \lambda_{j}}} y^{\prime}}
\end{gathered}
$$

where FT is the Fourier Transform operator applied to the brightness distribution of the asteroid $O^{\prime},\left(x^{\prime}, y^{\prime}\right)$ is a system of coordinates rotated by an angle $P A$ with respect to the $(x, y)$ frame. The $y^{\prime}$ axis thus coincides with the direction 
of the baseline (see Fig. 2) so that we replace the baseline vector, noted $\mathbf{B}$, by its modulus, noted $B$, in the expression of the model visibility above. $N_{y^{\prime}}$ is the number of pixels in the image along the $y^{\prime}$ axis. In Eqs. 4 and 5 , we have explicitly written the dependence of the flux and the visibility on the mesh scale factor $a$. It is important to note that changing the scale of an object by a linear factor $a$ is equivalent to multiply the baseline length by a factor $a$, or inversely.

[Fig. 2 here]

The free parameters of the TPM are: size (which is varied through the scale factor $a$ ), thermal inertia, and macroscopic surface roughness. The fit procedure involves calculation of $I_{i}^{\prime}\left(\lambda_{j}\right)$ and $V_{i}^{\prime}\left(B / \lambda_{j}\right)$ at each observing epoch and each wavelength $\lambda_{j}$, for a number of discrete values of $\Gamma$ and macroscopic surface roughness. Note that the dependence of the $\bar{\chi}^{2}$ on $a$ is trivial, as it can be seen from Eqs. 4 and 5. Thus, the best value of $a$ for each discrete value of $\Gamma$ and each roughness model can be found by minimizing the Eq. 2, Then, the location of the minimum $\bar{\chi}^{2}$ as function of $\Gamma$ gives the best-fit asteroid surface thermal inertia for each roughness model. Eventually, the value of $a$ at $\Gamma$-minimum is used to determine the best-fit value of $D_{\vee}$.

In some cases, the correction to the rotational phase $\Delta_{\phi}$ has to be treated as a free parameter of the TPM. In this case, the location of the minimum $\bar{\chi}^{2}$, as a function of $\Delta_{\phi}$ for different roughness and thermal inertia values, gives the set of best-fit physical parameters for the asteroid. An optical lightcurve obtained quasi-simultaneously could help reduce $\Delta_{\phi}$.

In the next section, we describe our observations of the asteroid (41) Daphne and the related data reduction. 


\section{Observations and data reduction}

The observations of (41) Daphne were carried out in service mode, on 2008 March 12 and 14 . We adopted the typical observing sequence with MIDI, which is extensively described by Przygodda et al. (2003). Using two ATs in the E0-G0 baseline configuration $(B=16 \mathrm{~m})$, we acquired four visibility observations for (41) Daphne, two on March 12 and two on March 14. TableL2 reports the observational circumstances. The telescopes and the delay lines of the interferometer were tracked at the rates predicted from the ephemerides of the target.

[Table. 2 here]

Our observations included a mid-infrared photometric and interferometric calibrator chosen from the ESO database, namely HD123139. The absolutely calibrated infrared spectrum of the calibration star was taken from Cohen (1999). Extraction and calibration of the visibility measurements of (41) Daphne were performed using the same method as Delbo et al. (2009). The flux and visibility measurements of (41) Daphne are shown in Fig[3 (or identically in Fig 4). The estimation of error bars constitutes a difficult issue when reducing MIDI data. In the most common case, when the 'high-sensitivity' mode is used, the photometry is acquired about 3 to 6 minutes after the fringes are recorded. Therefore, the measured value of $I(\lambda)$ does not correspond to the flux of the source at the time of the fringes recording. This leads to typical uncertainties of about 10 to $15 \%$ (see Chesneau, 2007, for more details). This error strongly depends on the atmospheric conditions during the night. An estimation of the error bars can thus be obtained by computing the RMS of the visibilities values of several calibrators observed closely to the source (or identically several observations of the same calibrator). We used this procedure for the two first visibilities, and considered a typical relative uncertainty of $15 \%$ for the two others since only one calibrator was observed during the second night.

[Fig. 3] and Fig. 4 here] 
In addition to the interferometric observations, we obtained an optical lightcurve of (41) Daphne, in order to better constrain the rotational phase at the epoch of the VLTI observations. In October 2009, during the tests of the $0.4 \mathrm{~m}$ telescope for the Antarctic Search for Transiting ExoPlanets (ASTEP) project (Daban et al., 2010) at the Observatoire de la Côte d'Azur in Nice, France, we performed time-resolved CCD photometric observations of this asteroid in the visible. Our observations spanned a period of about $3 \mathrm{~h}$. We used the technique of differential photometry, which makes use of stars present in the same CCD frame as the target, to allow for accurate removal of systematic effects such as atmospheric extinction variability. The telescope was tracked at the sidereal rate. The reduction of the $\mathrm{CCD}$ frames consisted in the conventional bias removal and flat-fielding, which was performed by using bias and flat calibration frames. The corresponding lightcurve is shown in Fig. 5 , where the times of the observations were light-travel subtracted.

\section{[Fig. 5] here]}

In the next section we describe the different shape models used for (41) Daphne, and how the TPM analysis was performed using this information).

\section{Shape models and TPM analysis}

For sake of comparison and evaluation of the results variability, two shape models were used in this study:

1. a convex mesh was downloaded (March 2010) from the Database of Asteroid Models from Inversion Techniques or DAMIT (see Durech et al., 2010, and http://astro.troja.mff.cuni.cz/projects/asteroids3D/web.php?page=project_main_page). This is a database of three-dimensional models of asteroids derived by solving the inverse problem of determining the object's shape and its rotational state from optical lightcurves. It also lists some models derived 
from a combination of adaptive optics images and lightcurves. The convex shape of (41) Daphne contains 1022 vertexes and 2040 triangular planar facets. The pole solution is also given: $\lambda_{0}=198^{\circ} \beta_{0}=-32^{\circ}, \mathrm{P}=5.98798 \mathrm{~h}$, $\phi_{0}=0^{\circ}$ at the reference epoch $t_{0}=2444771.79382(\mathrm{JD})$;

2. the second shape model of (41) Daphne, provided by B. Carry (private communication) 3 , is non-convex and was derived from the KOALA method (Carry et al., 2010; Kaasalainen, 2011). We recall here that this method allows one to derive the size and the shape of an asteroid from the inversion of data obtained by different observational techniques: namely, photometric lightcurves and adaptive optics images in the case of (41) Daphne. The corresponding pole solution is: $\lambda_{0}=198^{\circ} \beta_{0}=-31^{\circ}, \mathrm{P}=5.987980 \mathrm{~h}, \phi_{0}=0^{\circ}$ at the reference epoch $t_{0}=2444771.79382(\mathrm{JD})$.

Note that, while the size of the first shape model is arbitrary, the non-convex shape, derived from the KOALA method, has a volume equivalent diameter of $D_{\vee}=185 \pm 5 \mathrm{~km}$. We call it 'the nominal size' in the following. The pole solution of the convex model of (41) Daphne was obtained from inversion of a set of 23 optical lightcurves obtained in the period 1976 - 1988. Assuming $t_{0}=$ December 21, 1988 and $\sigma_{P}=0.00001$ hours, we thus find that $\Delta_{\phi} \sim 17^{\circ}$ at the epoch of our VLTI observations, given the rotation period of 5.98798 hours for this object. As a consequence, we attempted to better constrain the rotational phase of (41) Daphne using our recently obtained lightcurve shown in Fig. 5 , To do so, we calculated synthetic visible lightcurves using the convex shape of (41) Daphne and attempted to fit the model to the observed lightcurve using the value of $\Delta_{\phi}$ as a free parameter. A geometric scattering model was adopted: namely the visible reflected flux from each facet is proportional to the projected area of the facet, and it is required that the facet is also illuminated by the sun. Fig. 5 also shows the best fit model lightcurve that we obtained using the convex model for a correction value of $\Delta_{\phi}=(-0.2 \pm 0.3)^{\circ}$. The correction to

\footnotetext{
${ }^{3}$ See also his PHD thesis manuscript available at http://benoit.carry.free.fr/ for further information.
} 
the nominal rotational phase is thus negligible.

For the non-convex model, given that the KOALA model uses recent observations from 2008 March 28, and that the period is more accurate (by an order of magnitude), the error on the rotational phase given by the pole solution is negligible as well. As a consequence, for the two shape models, the rotational phase was not treated as a free parameter. The TPM was run for each roughness model (see Table 1), and each value of thermal inertia, namely 0, 5, 10, 25, 50, $75,100,150,200,300,400$ and $500 \mathrm{~J} \mathrm{~m}^{-2} \mathrm{~s}^{-0.5} \mathrm{~K}^{-1}$. Then the fit procedure described in section 2 was applied to the measured fluxes and visibilities.

In the next section we describe and discuss the results obtained from the application of the TPM to the observed visibilities and fluxes of (41) Daphne.

\section{Results}

\subsection{Convex shape model}

Fig. 6] shows our best-fit estimator $\bar{\chi}^{2}$ as a function of $\Gamma$ for the four different roughness models, in the case of the convex shape.

\section{[Fig. 6 here]}

We note that a surface with a low or no macroscopic roughness and a value of thermal inertia $<100 \mathrm{~J} \mathrm{~m}^{-2} \mathrm{~s}^{-0.5} \mathrm{~K}^{-1}$ gives the best fit to the observations. In particular the minima of the 'no roughness' and 'low roughness' models are at $\Gamma=48$ and $8 J m^{-2} s^{-0.5} K^{-1}$, respectively. The corresponding values of $D_{\vee}$ are 209 and $194 \mathrm{~km}$, with associated $p_{V}$ values of 0.057 and 0.067 , respectively. In Fig. 3 we plot the visibility and flux of the best-fit model ('no roughness', $\Gamma=$ $\left.48 J \mathrm{~m}^{-2} \mathrm{~s}^{-0.5} \mathrm{~K}^{-1}, D_{\vee}=209 \mathrm{~km}, p_{V}=0.057\right)$, in addition to the measured fluxes and visibilities of (41) Daphne. We note that our model represents well the observed flux except for the third observing epoch, where the flux of the TPM is greater than the measured one (by roughly $20 \%$ at $13 \mu \mathrm{m}$ ). This 'offsetlike' mismatch may likely come from an underestimation of the source flux by 
MIDI, especially around $13 \mu \mathrm{m}$ where the signal to noise ratio is generally quite low (Chesneau, 2007). The photometry measurement of the source may thus be degraded by a bad estimation and suppression of the thermal background (and its fluctuations), which is dominant in the mid-infrared (see e.g., Perrin et al., 2003). Moreover, the underestimation of the flux at the third epoch implies a similar offset-like mismatch between the corresponding measured and calculated visibilities at the same epoch.

In order to estimate the statistical uncertainty affecting the fit parameters $\Gamma$ and $D_{\vee}$, a Monte-Carlo analysis was performed. To this end, 200 normally distributed flux and visibility values per observation were generated at each wavelength, with average and standard deviation matching the measured fluxes and visibilities within their respective $1-\sigma$ uncertainty. For each set of fluxes and visibilities, a fit of the model, as described in section [2, was performed. Then, we took the standard deviation of the $\Gamma$ and $D_{\vee}$ values at the minimum $\bar{\chi}^{2}$ as the $1-\sigma$ uncertainty on our best fit values of thermal inertia and volume equivalent diameter. As a result we find $\Gamma=48 \pm 5 \mathrm{~J} \mathrm{~m}^{-2} \mathrm{~s}^{-0.5} \mathrm{~K}^{-1}$ and $D_{\vee}=$ $209 \pm 1 \mathrm{~km}$ as the best fit solution for a model using the convex shape without roughness; and $\Gamma=8 \pm 8 J \mathrm{~m}^{-2} s^{-0.5} K^{-1}$ and $D_{\vee}=194 \pm 2 \mathrm{~km}$ as the best fit solution for a model using the convex shape with a low roughness.

\subsection{Non-convex shape model}

Figure 7 shows our best-fit estimator $\bar{\chi}^{2}$ of the TPM, calculated in the case of the non-convex shape.

[Fig. 7 here]

In this case, a model with a low or medium roughness and a thermal inertia $<100 \mathrm{~J} \mathrm{~m} \mathrm{~m}^{-2} \mathrm{~s}^{-0.5} \mathrm{~K}^{-1}$ gives the best fit to the observations. In particular, the minima of the 'low roughness' and 'medium roughness' models are at $\Gamma=9$ and 0 $J m^{-2} s^{-0.5} K^{-1}$, respectively. The corresponding value of $D_{\vee}$ are $189 \mathrm{~km}$ and $182 \mathrm{~km}$, respectively, with $p_{V}=0.07$. The solution with $\Gamma=0 \mathrm{~J} \mathrm{~m}^{-2} \mathrm{~s}^{-0.5} \mathrm{~K}^{-1}$ is 
non physical and thus we reject it. Although $100 \mathrm{~km}$-sized Main Belt asteroids are known to have low thermal inertia, their values are in general larger than $10 J \mathrm{~m}^{-2} \mathrm{~s}^{-0.5} \mathrm{~K}^{-1}$ (see e.g., Mueller and Lagerros, 1998, and references therein). We also used the Monte-Carlo procedure described above to estimate the uncertainties on the fit parameters. These uncertainties result to be of $1 J \mathrm{~m}^{-2} \mathrm{~s}^{-0.5} \mathrm{~K}^{-1}$ for the thermal inertia and $1 \mathrm{~km}$ for the volume equivalent diameter. We show in Fig. 4 the measured fluxes and visibilities of (41) Daphne along with the best fit of the non-convex case ('low roughness', $\left.\Gamma=9 J \mathrm{~m}^{-2} \mathrm{~s}^{-0.5} \mathrm{~K}^{-1}, D_{\vee}=189 \mathrm{~km}, p_{V}=0.070\right)$. Our model, when used with the non-convex shape, also provides a good match to the observed flux. We note that the flux prediction at the third epoch is as well higher than the measured one. This effect may also be due to an underestimation of the source flux by MIDI, as described above. Moreover, the non-convex model seems to better fit the measured visibilities than the convex one. This result is consistent with the fact that the non-convex shape model corresponds to a more realistic and detailed representation of (41) Daphne, as previously indicated by B. Carry (private communication) 3 who better reproduced an occultation profile of (41) Daphne with this shape model. We can note however a slight mismatch between the model predictions and the measurements at the fourth epoch. The mismatch is located around $\frac{B}{\lambda} \approx 6$ as $^{-1}$ (or $\lambda \approx 9.6 \mu \mathrm{m}$ ) i.e. close to the atmospheric ozone absorption feature. This time-dependent absorption feature can cause fluctuations and lower the signal to noise ratio on both the coherent flux and the photometry. Due to its relative variability, this feature is frequently imperfectly removed during the reduction process of interferometric observations (see e.g., Tubbs et al., 2004). All the results are summarized in Table 3.

\section{[Table 3 here]}




\section{Discussion}

\subsection{Size and geometric visible albedo}

The best-fit value of $D_{\vee}$ obtained from our TPM analysis of MIDI data using the non-convex shape model, namely $189 \pm 1 \mathrm{~km}$, presents a discrepancy with the nominal value $(185 \pm 5 \mathrm{~km})$ of about $4 \mathrm{~km}$. This is about 4 times greater than the statistical uncertainties implied by the photometric and visibilities measurements and estimated via our Monte Carlo method. However, this discrepancy represents a relative accuracy of $2 \%$ on the diameter that appears remarkably good for a typical size determination of asteroids. If the two equally plausible solutions in terms of our best-fit indicator, i.e. low roughness - $\Gamma=9 J m^{-2} s^{-0.5} K^{-1}$ and medium roughness $-\Gamma=0 J m^{-2} s^{-0.5} K^{-1}$, are considered in order to estimate the model error on the value of $D_{\vee}$, we found that the latter is $7 \mathrm{~km}$ i.e. $4 \%$ in relative accuracy.

When the convex model is used as an a priori shape for the TPM, the value of $D_{\vee}$ resulting from the analysis of MIDI data is between 194 and $209 \mathrm{~km}$ (with a mean value of about $200 \mathrm{~km}$ ), depending on whether a low-roughness or a noroughness model, respectively, is assumed for the surface of Daphne. Because both solutions are equally good in terms of our best-fit estimator, it is clear that the systematic model uncertainty dominates the final size determination. This systematic relative uncertainty is of the order of $7 \%$ on the diameter.

It is also evident that the best fit values of the equivalent volume diameter obtained when using the convex shape are larger than the nominal value of $D_{\vee}$ of the non convex shape. This is because the latter has a smaller volume (due to the presence of topographic concavities) for a similar projected size on the plane of the sky. This volume overestimation when the convex shape is used, clearly indicates that the imposed condition of convexity, imposed by the lightcurve inversion technique (see Kaasalainen and Torppa, 2001), introduces a significant systematic bias on the size when large concavities are present on the asteroid surface.

The geometric visible albedo values derived from the TPM with the convex 
and non-convex shapes are in the range between 0.05 and 0.07 . This is in agreement with the C-type taxonomic classification of (41) Daphne.

\subsection{Thermal properties}

We obtained for the first time good constraints on the determination of macroscopic roughness for both shape models, although the results slightly differ. While a very low roughness is preferred in the convex shape case, a low or moderate roughness gives the best-fit in the case of the non-convex one. We estimate that the corresponding mean surface slope values should lie between 10 and $29^{\circ}$ for (41) Daphne. A high macroscopic roughness is discarded, which is quite surprising given the important surface roughness expected for large asteroids. Large main-belt asteroids such as (1) Ceres and (2) Pallas are expected to have rough surfaces as found by Spencer et al. (1989) from the analysis of the thermal infrared emission of these bodies. It is also known that the observed zero-phase thermal emission of the Moon is well reproduced by a rough-surface model, i.e. $\rho_{c}=0.64$ and $\gamma_{c}=90^{\circ}$ (Spencer, 1990$)$. The possibility to constrain the macroscopic roughness in the thermal infrared is very interesting because it allows, in principle, a more accurate determination of the asteroids thermal inertia from TPM modeling.

Our TPM analysis indicates that (41) Daphne has a thermal inertia value certainly smaller than $50 \mathrm{~J} \mathrm{~m}^{-2} \mathrm{~s}^{-0.5} \mathrm{~K}^{-1}$, and likely in the range between 10 and $\sim 30 \mathrm{~J} \mathrm{~m} \mathrm{~m}^{-2} \mathrm{~s}^{-0.5} \mathrm{~K}^{-1}$, indicating that a layer of very fine regolith covers its surface. This is in agreement with the thermal inertia values already measured on main-belt asteroids larger than $100 \mathrm{~km}$ in diameter. Indeed, from mid- and far-infrared observations, Mueller and Lagerros (1998) derived very low thermal inertia values, between 5 and $25 \mathrm{~J} \mathrm{~m}^{-2} \mathrm{~s}^{-0.5} \mathrm{~K}^{-1}$, for some large Main Belt asteroids including (1) Ceres, (2) Pallas, (3) Juno, (4) Vesta, and (532) Herculina. Moreover, Müller and Blommaert (2004) derived a thermal inertia of about $15 \mathrm{~J} \mathrm{~m} \mathrm{~m}^{-2} \mathrm{~s}^{-0.5} \mathrm{~K}^{-1}$ for (65) Cybele. More recently, from the direct measurement of the cooling during shadowing events in a binary system, Mueller et al. (2010) measured a thermal inertia of $20 \pm 15 \mathrm{~J} \mathrm{~m}^{-2} \mathrm{~s}^{-0.5} \mathrm{~K}^{-1}$ for 
the large binary Trojan $(D \approx 100 \mathrm{~km}),(617)$ Patroclus.

Several works were recently devoted to the determination of the thermal inertia value of the asteroids surface from TPM analysis of disk integrated thermal infrared data (see e.g., Delbo et al., 2007; Delbo and Tanga, 2009; Mueller, 2007, and references therein). In general, these works clearly showed that a good fit of the model to the observations can be obtained by using any roughness model. Because the determination of the value of the thermal inertia is a function of the roughness model adopted, any constraint on the latter parameter will allow improving the determination of thermal inertia. In order to show the effect of the visibility on the determination of a simultaneous solution for both thermal inertia and surface roughness, we also performed a TPM analysis of the disk integrated thermal infrared data of (41) Daphne, using the non-convex shape and neglecting the visibility measurements.

\section{[Fig. 8 here]}

As it can be seen from Fig. 8, any roughness model and any value of the thermal inertia give a value of $\bar{\chi}^{2}$ smaller than 1 , which implies that any value of these parameters allows the TPM to fit equally well the infrared flux. In order to reduce this degeneracy, it is known that thermal infrared photometric observations are needed at different illuminations and viewing geometries in order to constrain the asteroids thermal properties from a TPM modeling of photometric observations only.

\section{Conclusion}

We have obtained the first successful interferometric observations of asteroids in the thermal infrared using the ATs of the ESO VLTI. We observed the asteroid (41) Daphne using the MIDI instrument and the 16m-long baseline E0-G0.

We developed a thermophysical model (TPM) for the analysis of interfero- 
metric observations of asteroids in the thermal infrared, with the aim of deriving information about size and thermal properties.

We applied our TPM to the MIDI observations of (41) Daphne: our results indicate that Daphne has a volume equivalent diameter between 194 and 209 $\mathrm{km}$, depending on the surface roughness, if a convex shape model derived from lightcurve inversion is used as an a priori constraint on the shape of the asteroid. Since the nominal size, attached to the non-convex KOALA model, is $185 \pm 5 \mathrm{~km}$ (B. Carry, private communication) 3 , our results confirm that the assumption of convexity introduces a systematic bias on the size determination when important concavities are present on the asteroid's surface. In contrast, if the non-convex shape model is used, the volume equivalent diameter obtained from the TPM is $189 \pm 1 \mathrm{~km}$, i.e. very close to the nominal value of $185 \pm 5 \mathrm{~km}$. We estimated our model systematic uncertainty to be of $4 \%$ and of $7 \%$ on the determination of the asteroid volume equivalent diameter depending on whether the non-convex or the convex shape is used, respectively.

Our TPM analysis also showed that the macroscopic surface roughness can be constrained by interferometry, thanks to the angular resolving power offered by the VLTI and which allows to resolve the temperature distribution on the asteroid surface. In particular, using both shape models of (41) Daphne, we found a moderate to low roughness (see Table 1); 'high roughness' models are discarded by our analysis. With such a constraint on the macroscopic roughness, the TPM results indicate a very low thermal inertia for (41) Daphne, certainly smaller than $50 \mathrm{~J} \mathrm{~m}^{-2} \mathrm{~s}^{-0.5} \mathrm{~K}^{-1}$. This confirmed previous results indicating that the surface of asteroids with sizes larger than $100 \mathrm{~km}$ has a low thermal inertia. As shown by this work, the possibility of constraining the macroscopic roughness is important in the prospect of an accurate modelling of the thermal infrared emission of asteroids, and especially thermal inertia determination (see also Mueller, 2007; Delbo and Tanga, 2009). 


\section{Acknowledgments}

We would like to thank the staff and the Science Archive Operation of the European Southern Observatory (ESO) for their support in the acquisition of the data. The comments from the referee Benoit Carry and an anonymous referee were extremely helpful in revising this work and allowed us to improve significantly the manuscript. 


\section{References}

Bottke, W.F., Durda, D.D., Nesvorný, D., Jedicke, R., Morbidelli, A., Vokrouhlický, D., Levison, H.F., 2005. Linking the collisional history of the main asteroid belt to its dynamical excitation and depletion. Icarus 179, 63-94.

Bottke, Jr., W.F., Vokrouhlický, D., Rubincam, D.P., Nesvorný, D., 2006. The Yarkovsky and Yorp Effects: Implications for Asteroid Dynamics. Annu. Rev. Earth Planet. 34, 157-191.

Bowell, E., Hapke, B., Domingue, D., Lumme, K., Peltoniemi, J., Harris, A.W., 1989. Application of photometric models to asteroids, in: R. P. Binzel, T. Gehrels, \& M. S. Matthews (Ed.), Asteroids II, pp. 524-556.

Carry, B., Dumas, C., Kaasalainen, M., Berthier, J., Merline, W.J., Erard, S., Conrad, A., Drummond, J.D., Hestroffer, D., Fulchignoni, M., Fusco, T., 2010. Physical properties of (2) Pallas. Icarus 205, 460-472.

Chesneau, O., 2007. MIDI: Obtaining and analysing interferometric data in the mid-infrared. New Astron. Rev. 51, 666-681.

Christensen, P.R., Bandfield, J.L., Bell, J.F., Gorelick, N., Hamilton, V.E., Ivanov, A., Jakosky, B.M., Kieffer, H.H., Lane, M.D., Malin, M.C., McConnochie, T., McEwen, A.S., McSween, H.Y., Mehall, G.L., Moersch, J.E., Nealson, K.H., Rice, J.W., Richardson, M.I., Ruff, S.W., Smith, M.D., Titus, T.N., Wyatt, M.B., 2003. Morphology and Composition of the Surface of Mars: Mars Odyssey THEMIS Results. Science 300, 2056-2061.

Cohen, J.G., 1999. The Spectra of Main-Sequence Stars in Galactic Globular Clusters. II. CH and CN Bands in M71. Astron. J. 117, 2434-2439.

Daban, J.B., Gouvret, C., Guillot, T., Agabi, A., Crouzet, N., Rivet, J.P., Mekarnia, D., Abe, L., Bondoux, E., Fanteï-Caujolle, Y., Fressin, F., Schmider, F.X., Valbousquet, F., Blanc, P.E., Le van Suu, A., Rauer, H., Erikson, A., Pont, F., Aigrain, S., 2010. ASTEP 400: a telescope designed 
for exoplanet transit detection from Dome C, Antarctica, in: Society of PhotoOptical Instrumentation Engineers (SPIE) Conference Series, volume 7733. p. $77334 \mathrm{~T}$.

Delbo, M., Dell'Oro, A., Harris, A.W., Mottola, S., Mueller, M., 2007. Thermal inertia of near-Earth asteroids and implications for the magnitude of the Yarkovsky effect. Icarus 190, 236-249.

Delbo, M., Harris, A.W., 2002. Physical properties of near-Earth asteroids from thermal infrared observations and thermal modeling. Meteor. and Plan. Sci. 37, 1929-1936.

Delbo, M., Ligori, S., Matter, A., Cellino, A., Berthier, J., 2009. First VLTIMIDI Direct Determinations of Asteroid Sizes. Astrophys. J. 694, 1228-1236.

Delbo, M., Tanga, P., 2009. Thermal inertia of main belt asteroids smaller than $100 \mathrm{~km}$ from IRAS data. Plan. and Space Sci. 57, 259-265.

Descamps, P., Marchis, F., Pollock, J., Berthier, J., Vachier, F., Birlan, M., Kaasalainen, M., Harris, A.W., Wong, M.H., Romanishin, W.J., Cooper, E.M., Kettner, K.A., Wiggins, P., Kryszczynska, A., Polinska, M., Coliac, J., Devyatkin, A., Verestchagina, I., Gorshanov, D., 2008. New determination of the size and bulk density of the binary Asteroid 22 Kalliope from observations of mutual eclipses. ICARUS 196, 578-600.

Durech, J., Sidorin, V., Kaasalainen, M., 2010. DAMIT: a database of asteroid models. Astron. and Astrophys. 513, A46.

Emery, J.P., Sprague, A.L., Witteborn, F.C., Colwell, J.E., Kozlowski, R.W.H., Wooden, D.H., 1998. Mercury: Thermal Modeling and Mid-infrared (5-12 $\mu \mathrm{m})$ Observations. Icarus 136, 104-123.

Giorgini, J.D., Ostro, S.J., Benner, L.A.M., Chodas, P.W., Chesley, S.R., Hudson, R.S., Nolan, M.C., Klemola, A.R., Standish, E.M., Jurgens, R.F., Rose, R., Chamberlin, A.B., Yeomans, D.K., Margot, J.L., 2002. Asteroid 1950 
DA's Encounter with Earth in 2880: Physical Limits of Collision Probability Prediction. Science 296, 132-136.

Hapke, B., 1984. Bidirectional reflectance spectroscopy. III - Correction for macroscopic roughness. Icarus 59, 41-59.

Harris, A.W., Lagerros, J.S.V., 2002. Asteroids in the Thermal Infrared. Asteroids III , 205-218.

Hestroffer, D., Tanga, P., Cellino, A., Guglielmetti, F., Lattanzi, M., Di Martino, M., Zappalà, V., Berthier, J., 2002. Asteroids observations with the Hubble Space Telescope. I. Observing strategy, and data analysis and modeling process. Astron. and Astrophys. 391, 1123-1132.

Jakosky, B.M., 1986. On the thermal properties of Martian fines. Icarus 66, $117-124$.

Kaasalainen, M., 2011. Maximum compatibility estimates and shape reconstruction with boundary curves and volumes of generalized projections. Inv. Prob. and Imag., submitted .

Kaasalainen, M., Mottola, S., Fulchignoni, M., 2002. Asteroid Models from Disk-integrated Data. Asteroids III , 139-150.

Kaasalainen, M., Torppa, J., 2001. Optimization Methods for Asteroid Lightcurve Inversion. I. Shape Determination. Icarus 153, 24-36.

Lagerros, J.S.V., 1998. Thermal physics of asteroids. IV. Thermal infrared beaming. Astron. and Astrophys. 332, 1123-1132.

Leinert, C., Graser, U., Przygodda, F., Waters, L.B.F.M., Perrin, G., Jaffe, W., Lopez, B., Bakker, E.J., Böhm, A., Chesneau, O., Cotton, W.D., Damstra, S., de Jong, J., Glazenborg-Kluttig, A.W., Grimm, B., Hanenburg, H., Laun, W., Lenzen, R., Ligori, S., Mathar, R.J., Meisner, J., Morel, S., Morr, W., Neumann, U., Pel, J.W., Schuller, P., Rohloff, R.R., Stecklum, B., Storz, C., 
von der Lühe, O., Wagner, K., 2003. MIDI - the $10 \mu \mathrm{m}$ instrument on the VLTI. Astrophys. and Space Sci. 286, 73-83.

Milani, A., Chesley, S.R., Sansaturio, M.E., Bernardi, F., Valsecchi, G.B., Arratia, O., 2009. Long term impact risk for (101955) $1999 \mathrm{RQ}_{36}$. Icarus 203, $460-471$.

Morbidelli, A., Vokrouhlický, D., 2003. The Yarkovsky-driven origin of nearEarth asteroids. ICARUS 163, 120-134.

Mouret, S., Hestroffer, D., Mignard, F., 2007. Asteroid masses and improvement with Gaia. Astron. and Astrophys. 472, 1017-1027.

Mueller, M., 2007. Surface properties of asteroids from mid-infrared observations and thermophysical modeling. Ph.D. thesis. Freie Universitaet Berlin.

Mueller, M., Marchis, F., Emery, J.P., Harris, A.W., Mottola, S., Hestroffer, D., Berthier, J., di Martino, M., 2010. Eclipsing binary Trojan asteroid Patroclus: Thermal inertia from Spitzer observations. Icarus 205, 505-515. 0908.4198,

Mueller, T.G., Lagerros, J.S.V., 1998. Asteroids as far-infrared photometric standards for ISOPHOT. Astron. and Astrophys. 338, 340-352.

Müller, T.G., Barnes, P.J., 2007. $3.2 \mathrm{~mm}$ lightcurve observations of (4) Vesta and (9) Metis with the Australia Telescope Compact Array. Astron. and Astrophys. 467, 737-747.

Müller, T.G., Blommaert, J.A.D.L., 2004. ¡ASTROBJ ¿65 Cybelei/ASTROBJ in the thermal infrared: Multiple observations and thermophysical analysis. Astron. and Astrophys. 418, 347-356. arXiv:astro-ph/0401458.

Nesvorný, D., Bottke, W.F., 2004. Detection of the Yarkovsky effect for mainbelt asteroids. Icarus 170, 324-342.

Ostro, S.J., Hudson, R.S., Nolan, M.C., Margot, J., Scheeres, D.J., Campbell, D.B., Magri, C., Giorgini, J.D., Yeomans, D.K., 2000. Radar Observations of Asteroid 216 Kleopatra. Science 288, 836-839. 
Perrin, G., Leinert, C., Graser, U., Waters, L.B.F.M., Lopez, B., 2003. MIDI, the $10 \mu \mathrm{m}$ interferometer of the VLT, in: G. Perrin \& F. Malbet (Ed.), EAS Publications Series, volume 6. p. 127.

Przygodda, F., Chesneau, O., Graser, U., Leinert, C., Morel, S., 2003. Interferometric Observation at Mid-Infrared Wave-lengths with MIDI. Astrophys. and Space Sci. 286, 85-91.

Spencer, J.R., 1990. A rough-surface thermophysical model for airless planets. ICARUS 83, 27-38.

Spencer, J.R., Lebofsky, L.A., Sykes, M.V., 1989. Systematic biases in radiometric diameter determinations. ICARUS 78, 337-354.

Stuart, J.S., Binzel, R.P., 2004. Bias-corrected population, size distribution, and impact hazard for the near-Earth objects. ICARUS 170, 295-311.

Tanga, P., Comito, C., Paolicchi, P., Hestroffer, D., Cellino, A., Dell'Oro, A., Richardson, D.C., Walsh, K.J., Delbo, M., 2009. Rubble-Pile Reshaping Reproduces Overall Asteroid Shapes. Astrophys. J. 706, L197-L202.

Tanga, P., Hestroffer, D., Berthier, J., Cellino, A., Lattanzi, M.G., di Martino, M., Zappalá, V., 2001. HST/FGS Observations of the Asteroid (216) Kleopatra. Icarus 153, 451-454.

Tanga, P., Hestroffer, D., Cellino, A., Lattanzi, M., Di Martino, M., Zappalà, V., 2003. Asteroid observations with the Hubble Space Telescope. II. Duplicity search and size measurements for 6 asteroids. Astron. and Astrophys. 401, 733-741.

Tedesco, E.F., Williams, J.G., Matson, D.L., Weeder, G.J., Gradie, J.C., Lebofsky, L.A., 1989. A three-parameter asteroid taxonomy. Astron. J. 97, 580-606.

Thomas, P.C., Joseph, J., Carcich, B., Veverka, J., Clark, B.E., Bell, J.F., Byrd, A.W., Chomko, R., Robinson, M., Murchie, S., Prockter, L., Cheng, A., Izenberg, N., Malin, M., Chapman, C., McFadden, L.A., Kirk, R., Gaffey, 
M., Lucey, P.G., 2002. Eros: Shape, Topography, and Slope Processes. Icarus $155,18-37$.

Thomas, P.C., Veverka, J., Bell, J.F., Clark, B.E., Carcich, B., Joseph, J., Robinson, M., McFadden, L.A., Malin, M.C., Chapman, C.R., Merline, W., Murchie, S., 1999. Mathilde: Size, Shape, and Geology. Icarus 140, 17-27.

Tubbs, R.N., Meisner, J.A., Bakker, E.J., Albrecht, S., 2004. Differential phase delay observations with VLTI-MIDI at N-band, in: W. A. Traub (Ed.), Society of Photo-Optical Instrumentation Engineers (SPIE) Conference Series, volume 5491. p. 588.

Veverka, J., Farquhar, B., Robinson, M., Thomas, P., Murchie, S., Harch, A., Antreasian, P.G., Chesley, S.R., Miller, J.K., Owen, W.M., Williams, B.G., Yeomans, D., Dunham, D., Heyler, G., Holdridge, M., Nelson, R.L., Whittenburg, K.E., Ray, J.C., Carcich, B., Cheng, A., Chapman, C., Bell, J.F., Bell, M., Bussey, B., Clark, B., Domingue, D., Gaffey, M.J., Hawkins, E., Izenberg, N., Joseph, J., Kirk, R., Lucey, P., Malin, M., McFadden, L., Merline, W.J., Peterson, C., Prockter, L., Warren, J., Wellnitz, D., 2001a. The landing of the NEAR-Shoemaker spacecraft on asteroid 433 Eros. Nature 413, 390-393.

Veverka, J., Thomas, P.C., Robinson, M., Murchie, S., Chapman, C., Bell, M., Harch, A., Merline, W.J., Bell, J.F., Bussey, B., Carcich, B., Cheng, A., Clark, B., Domingue, D., Dunham, D., Farquhar, R., Gaffey, M.J., Hawkins, E., Izenberg, N., Joseph, J., Kirk, R., Li, H., Lucey, P., Malin, M., McFadden, L., Miller, J.K., Owen, W.M., Peterson, C., Prockter, L., Warren, J., Wellnitz, D., Williams, B.G., Yeomans, D.K., 2001b. Imaging of Small-Scale Features on 433 Eros from NEAR: Evidence for a Complex Regolith. Science 292, $484-488$.

Yano, H., Kubota, T., Miyamoto, H., Okada, T., Scheeres, D., Takagi, Y., Yoshida, K., Abe, M., Abe, S., Barnouin-Jha, O., Fujiwara, A., Hasegawa, S., Hashimoto, T., Ishiguro, M., Kato, M., Kawaguchi, J., Mukai, T., Saito, 
J., Sasaki, S., Yoshikawa, M., 2006. Touchdown of the Hayabusa Spacecraft at the Muses Sea on Itokawa. Science 312, 1350-1353. 


\section{Tables and Table Captions}

\begin{tabular}{lccc}
\hline Roughness model & $\gamma_{c}$ & $\rho_{c}$ & $\bar{\theta}$ \\
\hline no roughness & $0^{\circ}$ & 0.0 & $0^{\circ}$ \\
low roughness & $45^{\circ}$ & 0.5 & $10^{\circ}$ \\
medium roughness & $68^{\circ}$ & 0.8 & $29^{\circ}$ \\
high roughness & $90^{\circ}$ & 1.0 & $58^{\circ}$ \\
\hline
\end{tabular}

Table 1: The four roughness models used in the application of the TPM to the MIDI data; $\gamma_{c}$ and $\rho_{c}$ respectively correspond to the crater opening angle and the crater density, while $\bar{\theta}$ is the corresponding mean surface slope according to the parameterization introduced by Hapke (1984) (see text and also Delbo et al., 2007, for further details).

\begin{tabular}{cccccccc}
\hline Date & $\mathrm{UT}$ & $r(\mathrm{AU})$ & $\Delta(\mathrm{AU})$ & $\alpha(\mathrm{deg})$ & $B(\mathrm{~m})$ & $P A(\mathrm{deg})$ & Tag \\
\hline 2008-03-12 & $05: 19: 14$ & 2.0866 & 1.1920 & 15.8 & 13.6 & 66.6 & $\mathrm{D}_{1}$ \\
$2008-03-12$ & $06: 24: 35$ & 2.0864 & 1.1917 & 15.8 & 15.4 & 70.6 & $\mathrm{D}_{2}$ \\
$2008-03-14$ & $04: 19: 27$ & 2.0834 & 1.1769 & 15.0 & 11.6 & 61.6 & $\mathrm{D}_{3}$ \\
$2008-03-14$ & $04: 32: 48$ & 2.0834 & 1.1769 & 15.0 & 12.2 & 63.4 & $\mathrm{D}_{4}$ \\
\hline
\end{tabular}

Table 2: Observational circumstances and interferometric parameters of the (41) Daphne observations. $r$ and $\Delta$ are the heliocentric and geocentric distances, respectively, while $\alpha$ is the solar phase angle. $B$ and $P A$ are respectively the length and the position angle of the baseline projected on sky. The last column contains a tag associated with each observation. 


\begin{tabular}{cccccc}
\hline Shape & Roughness model & $\bar{\chi}^{2}$ & $\begin{array}{c}\Gamma \\
\left(J m^{-2} s^{-0.5} K^{-1}\right)\end{array}$ & $\begin{array}{c}\mathrm{D}_{\vee} \\
(\mathrm{km})\end{array}$ & $p_{V}$ \\
\hline \multirow{2}{*}{ Convex } & No roughness & $3.5 \pm 0.3$ & $48 \pm 6$ & $209 \pm 1$ & $0.057 \pm 0.009$ \\
& Low roughness & $3.7 \pm 0.3$ & $8_{-8}^{+10}$ & $194 \pm 2$ & $0.067 \pm 0.011$ \\
Non-convex & Low roughness & $3.4 \pm 0.3$ & $9 \pm 1$ & $189 \pm 1$ & $0.070 \pm 0.011$ \\
\hline
\end{tabular}

Table 3: Results of the determination of physical properties of the asteroid (41) Daphne, using the TPM. The $\bar{\chi}^{2}$ is our best-fit estimator as described by Eq $2 \Gamma$ is the thermal inertia, $D \vee$ is the spherical volume equivalent diameter, and $p_{V}$ is the geometric visible albedo. The errors are within $1-\sigma$. 


\section{Figure captions and figures}

\section{Fig 1 caption :}

Image of the asteroid (41) Daphne created from the TPM, using the convex shape model (left image) and the non-convex one (right image). The gray level is proportional to the emitted thermal infrared flux.

\section{Fig 2 caption :}

Illustration of the geometric parameters involved in the calculation of the synthetic visibility and flux from a TPM image.

\section{Fig 3 caption :}

Left panels: measured thermal infrared fluxes (with error bars) between 8 and $13 \mu \mathrm{m}$ of (41) Daphne, and the corresponding best-fit synthetic infrared fluxes (solid lines) derived from the TPM in the case of the convex shape; right panels: measured interferometric visibilities plotted, in $\mathrm{N}$ band, as a function of angular frequency, and the corresponding synthetic visibilities of the TPM (solid lines). The best-fit model represented here is : 'no roughness', $\Gamma=48 \mathrm{~J} \mathrm{~m}^{-2} \mathrm{~s}^{-0.5} \mathrm{~K}^{-1}$. The tags $D_{1}, D_{2}, D_{3}$ and $D_{4}$ indicate the observing epoch in the chronological order (see Table 2).

\section{Fig 4 caption :}

As for Fig. 3, but the best-fit model represented here, in the case of the nonconvex shape, is : 'low roughness', $\Gamma=9 \mathrm{~J} \mathrm{~m}^{-2} \mathrm{~s}^{-0.5} \mathrm{~K}^{-1}$. The tags $D_{1}, D_{2}$, $D_{3}$ and $D_{4}$ indicate the observing epoch in the chronological order (see Table 2).

\section{Fig 5 caption :}

Diamonds: lightcurve obtained from CCD photometric observations in the visible of (41) Daphne in October 2009 during the tests of the ASTEP $0.4 \mathrm{~m}$ telescope at the Observatoire de la Côte d'Azur, Nice, France. These observations spanned a period of about 3 hours. Solid curve: corresponding best-fit lightcurve from the convex model.

\section{Fig 6 caption :}

Plot of $\bar{\chi}^{2}$ (see Eq. 21), calculated from the TPM in the case of the convex shape, as a function of thermal inertia $\Gamma$, for the four roughness models (see Table 1). 


\section{Fig 7 caption :}

Plot of $\bar{\chi}^{2}$ (see Eq. 21), calculated from the TPM in the case of the non-convex shape, as a function of thermal inertia $\Gamma$, for the four roughness models (see Table 1).

\section{Fig 8 caption :}

Plot of $\bar{\chi}^{2}$ (see Eq. 2) calculated from the TPM, using only the flux measurements of (41) Daphne. This is represented in the case of the non-convex shape, as a function of thermal inertia $\Gamma$, for the four roughness models (see Table 1). 

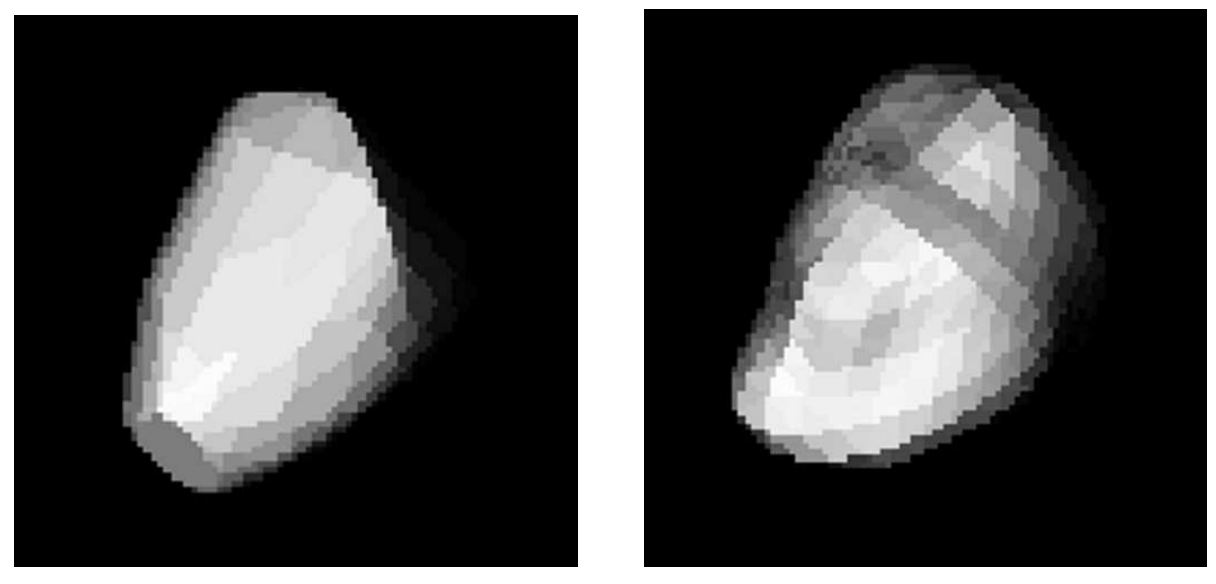

Figure 1: 


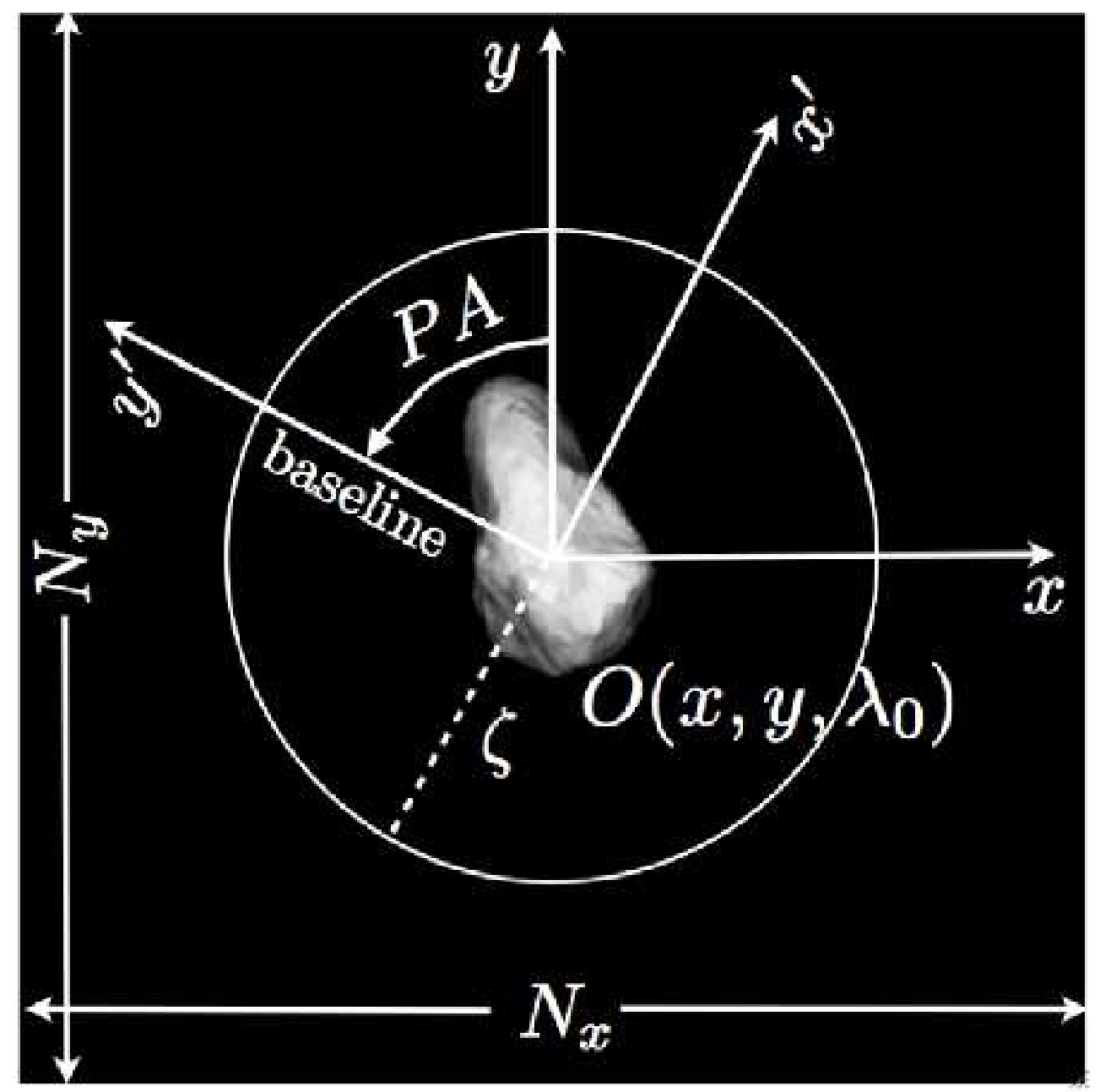

Figure 2: 

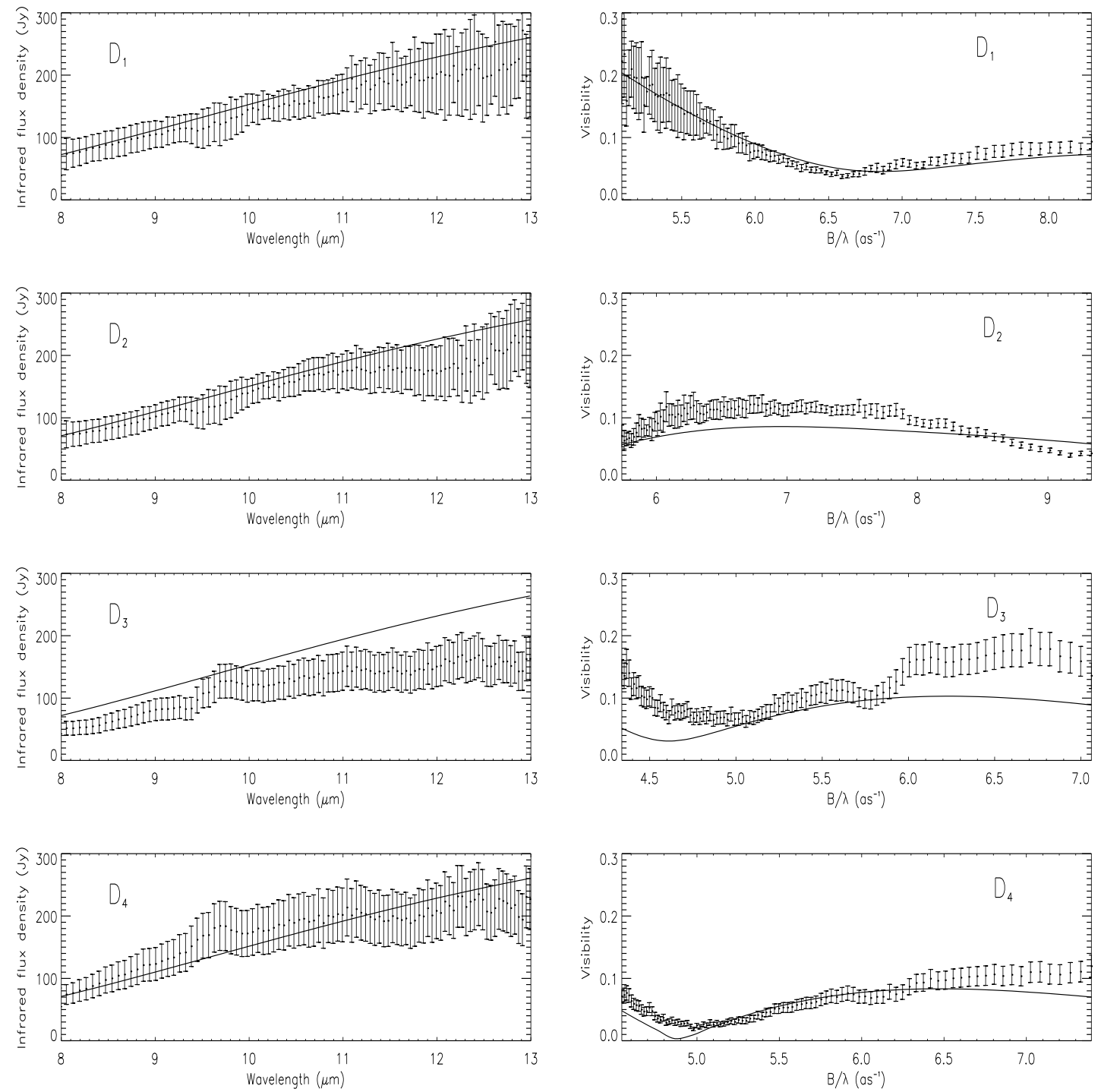

Figure 3: 

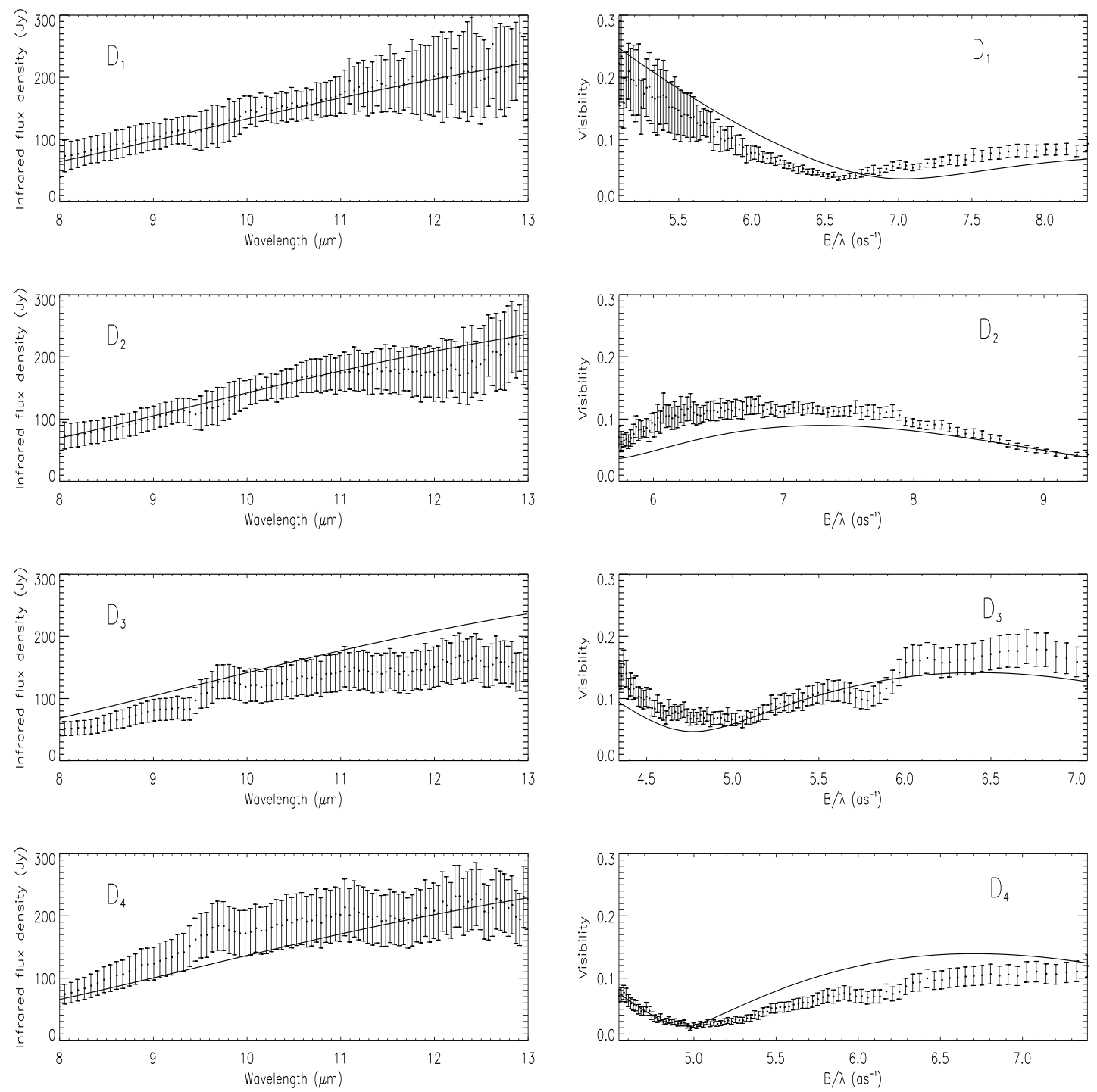

Figure 4: 


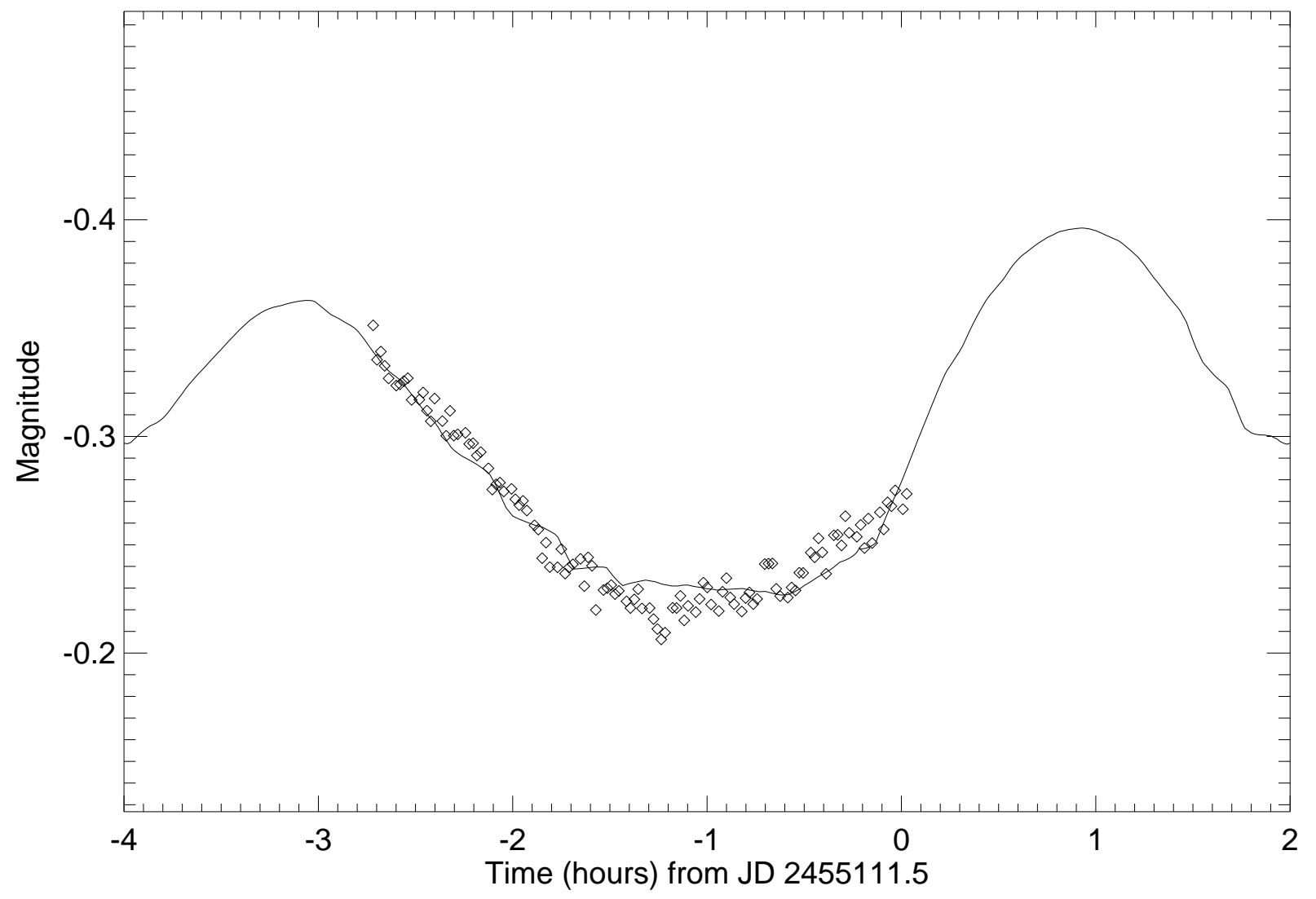

Figure 5: 


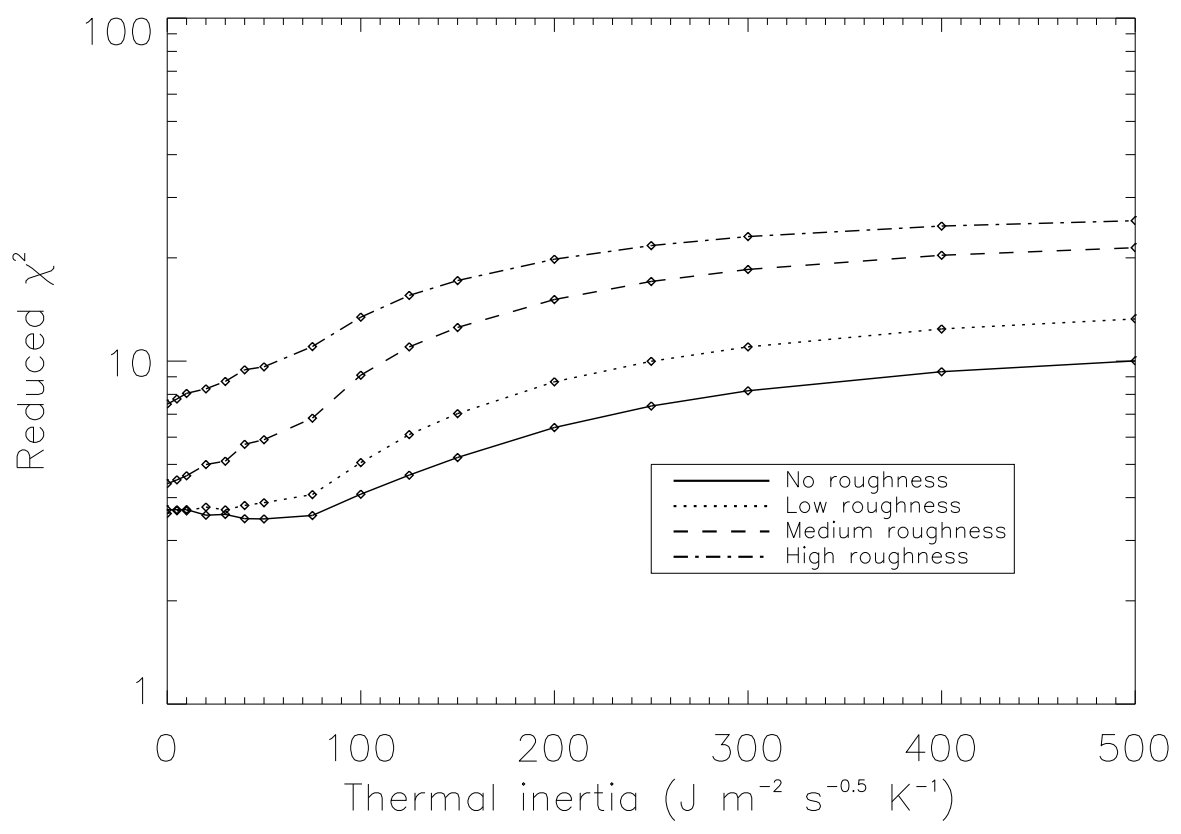

Figure 6: 


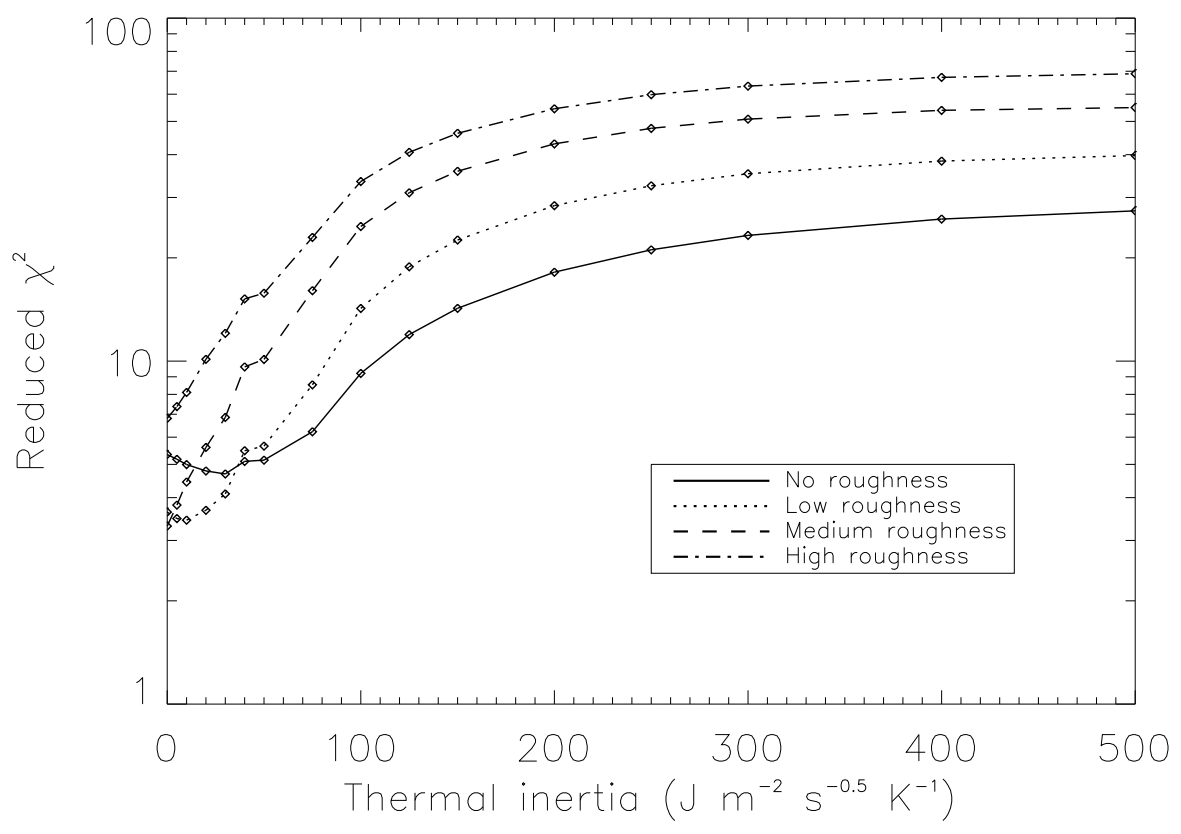

Figure 7: 


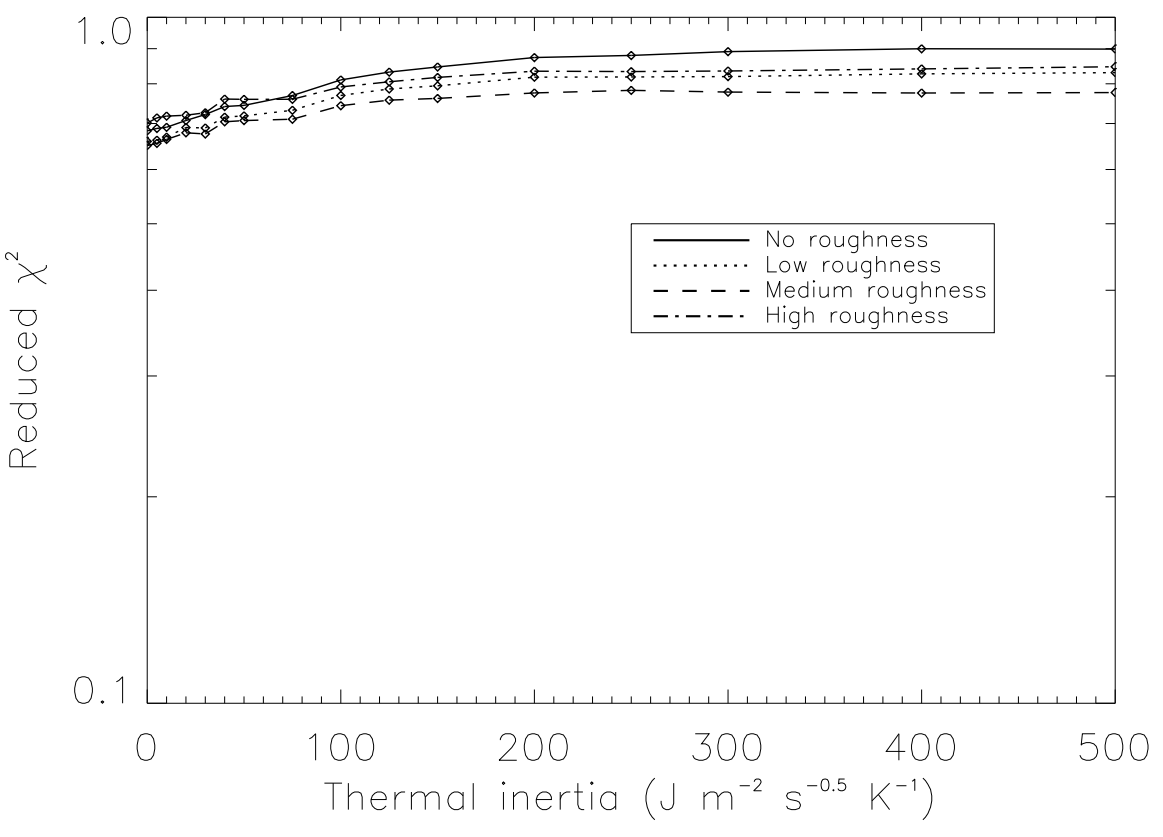

Figure 8: 